\title{
On two recent geometrical characterizations of hyperellipticity
}

\author{
Antonio F. Costa and Ana M. Porto \\ Departamento de Matemáticas Fundamentales \\ Facultad de Ciencias, UNED, \\ 28040 Madrid, Spain \\ acosta@mat.uned.es \\ asilva@mat.uned.es
}

Recibido: 9 de Septiembre de 2002

Aceptado: 8 de Enero de 2003

\begin{abstract}
We obtain short and unified new proofs of two recent characterizations of hyperellipticity given in [4] and [6], as well as a way of establishing a relation between them.

Key words: Riemann surface, hyperelliptic involution, Fuchsian group, hyperbolic polygon

2000 Mathematics Subject Classification: 30F20, 30F10
\end{abstract}

\section{Introduction}

We offer a new point of view of two recent geometrical characterizations of hyperellipticity of Riemann surfaces of genus $g \geq 2$ (see [4] and [6]). We introduce two special types of fundamental regions for surface Fuchsian groups, i. e. Fuchsian groups without elliptic elements uniformizing closed Riemann surfaces. With this approach, the Maskit characterization of hyperellipticity is equivalent to the rotational symmetry for a type of hyperbolic polygon that is a fundamental region for surface Fuchsian groups. The Schaller characterization of hyperellipticity is equivalent to the existence of a fundamental hyperbolic polygon for surface Fuchsian groups where the elements of the group induce the identification of opposite sides of the polygon. In this way, we obtain short and unified new proofs of the two recent characterizations of hyperellipticity and a way of establishing a relation between them. In order to have basic information about hyperelliptic Riemann surfaces see for instance [1] or [5].

The authors are partially supported by BFM2002-04801. 


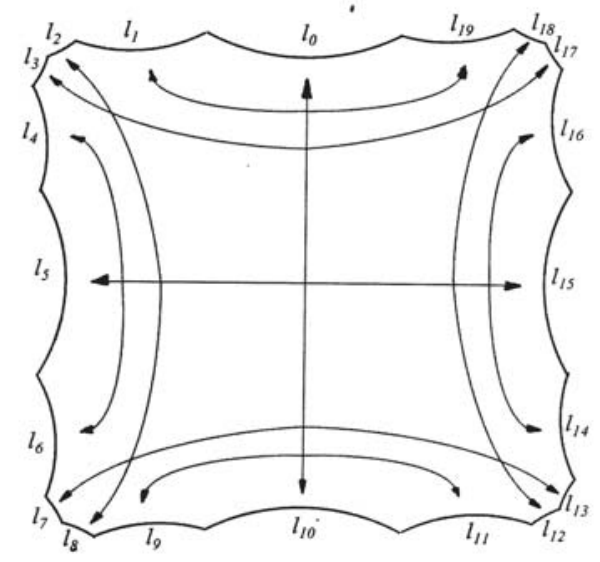

Figure 1: Prehyperelliptic polygon $(\mathrm{g}=3)$.

\section{Prehyperelliptic and centralhyperelliptic fundamental poly- gons for Fuchsian groups}

In this work we shall consider Riemann surfaces of genus $g \geq 2$ equipped with a metric of constant curvature -1 . We start by defining two special types of fundamental polygons for surface Fuchsian groups.

Definition 2.1 (Prehyperelliptic fundamental polygon). Let $\Gamma$ be a surface Fuchsian group of genus $g \geq 2$. A fundamental polygon $P$ for $\Gamma$ is said to be prehyperelliptic if and only if (see Figure 1):

(i) $P$ has $m=8 g-4$ sides with labelling $l_{\overline{0}}, l_{\overline{1}}, \ldots, l_{\overline{m-1}}$ where $\{\overline{0}, \overline{1}, \overline{2}, \ldots, \overline{m-1}\}=$ $\mathbb{Z} / m \mathbb{Z}$.

(ii) There are hyperbolic transformations in $\Gamma$ sending $l_{\overline{2 i}}$ to $l_{\frac{m}{2}-2 i}$ and $l_{\overline{2 i+1}}$ to $l-2 i-1$.

(iii) Let $\widehat{V}_{\bar{i}}$ denote the measure of the interior angle of $P$ with sides $l_{\bar{i}}$ and $l_{\overline{i+1}}$. The following conditions must be satisfied:

$$
\widehat{V}_{\bar{i}}=\widehat{V_{\overline{i+\frac{m}{2}}}}, \quad \widehat{V}_{\bar{i}}+\widehat{V_{-i-1}}=\pi, \quad \widehat{V}_{\bar{i}}+\widehat{V_{-i-1+\frac{m}{2}}}=\pi .
$$

Remark that by condition (iii) every prehyperelliptic fundamental polygon is convex. 


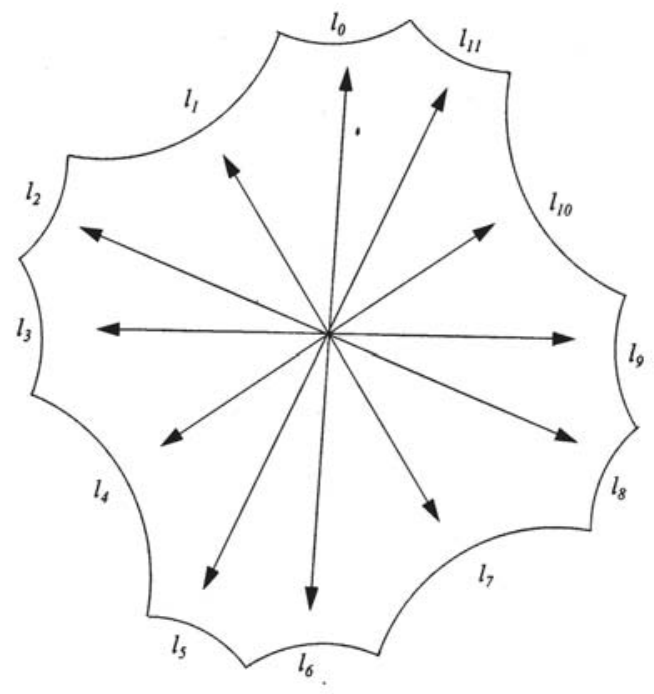

Figure 2: Centralhyperelliptic polygon $(\mathrm{g}=3)$.

Definition 2.2 (Centralhyperelliptic fundamental polygon). Let $\Gamma$ be a surface Fuchsian group of genus $g \geq 2$. A fundamental polygon $P$ for $\Gamma$ is said to be centralhyperelliptic if and only if (see Figure 2):

(i) $P$ has $4 g$ sides with labelling $l_{\overline{0}}, l_{\overline{1}}, \ldots, l_{\overline{4 g-1}}$, where $\{\overline{0}, \overline{1}, \overline{2}, \ldots, \overline{4 g-1}\}$ $=\mathbb{Z} / 4 g \mathbb{Z}$.

(ii) There are hyperbolic transformations in $\Gamma$ sending $l_{\bar{i}}$ to $l_{\overline{i+2 g}}, i=0, \ldots, 2 g-1$.

(iii) The interior angles of $P$ satisfy $\widehat{V}_{\bar{i}}=\widehat{V_{\overline{i+2 g}}}, V_{\bar{i}}=l_{\bar{i}} \cap l_{\overline{i+1}}, i=0, \ldots, 2 g-1$.

Since $\Gamma$ is a surface group remark that $\sum_{i=0}^{2 g-1} \widehat{V}_{\bar{i}}=2 \pi$.

Proposition 2.3. Every centralhyperelliptic fundamental polygon $P$ has rotational symmetry of order two, $i$. e. there is an order two elliptic transformation $r$ such that $r(P)=P$.

Proof. We recall the following well-known property about the congruency of hyperbolic polygons:

Let $P$ and $P^{\prime}$ be two hyperbolic polygons. Let $L V=\left\{l_{i}, V_{i}\right\}$ and $L V^{\prime}=\left\{l_{i}^{\prime}, V_{i}^{\prime}\right\}$ be the sets of sides and vertices of $P$ and $P^{\prime}$ respectively. Let $\phi: L V \rightarrow L V^{\prime}$ be a bijection 
sending vertices to vertices, sides to sides and preserving the adjacency relation. Let $W$ be a subset of $L V$ having three elements. Assume that length $\left(l_{i}\right)=\operatorname{length}\left(\phi\left(l_{i}\right)\right)$ and $\widehat{V}_{i}=\widehat{\phi\left(V_{i}\right)}$, where $\widehat{V}_{i}\left(\right.$ resp. $\left.\widehat{\phi\left(V_{i}\right)}\right)$ is measure of the interior angle of $P\left(\right.$ resp. $\left.P^{\prime}\right)$ with vertex in $V_{i}$ (resp. $\left.\phi\left(V_{i}\right)\right)$ and $l_{i}, V_{i} \in L V-W$. Then the bijection $\phi$ is induced by an hyperbolic transformation.

Now let $P$ be a centralhyperelliptic fundamental polygon of a surface Fuchsian group. Let $d$ be a diagonal joining two opposite vertices of $P$. The diagonal $d$ divides the polygon $P$ in two polygons $C_{1}$ and $C_{2}$. Let $r$ be the elliptic element of order two with fixed point the middle point of the diagonal $d$. Let $P^{\prime}$ be the polygon obtained by the union of $C_{1}$ and $r\left(C_{1}\right)$. The polygons $P$ and $P^{\prime}$ have the vertices of $C_{1}$ in common. We can establish a bijection $\phi$ between the sets of vertices and sides of $P$ and $P^{\prime}$ that preserves adjacency and fixes the sides and vertices of $C_{1}$. Then condition (ii) of the definition of centralhyperelliptic polygons implies that, if $l$ is a side in $P, l$ and $\phi(l)$ have the same measure. And if $V_{1}$ and $V_{2}$ are the vertices at the ends of $d$, it follows by condition (iii) that $\widehat{V}=\widehat{\phi(V)}, V$ different from $V_{1}$ and $V_{2}$. Therefore the polygons $P$ and $P^{\prime}$ are congruent and, since $C_{1}$ is common to $P$ and $P^{\prime}$, then $P$ and $P^{\prime}$ are the same polygon and thus $r$ is a rotational symmetry of order two of $P$.

The following result is the main Theorem of this work:

Theorem 2.4. Let $\Gamma$ be a surface Fuchsian group of genus $g \geq 2$. The following three conditions are equivalent:

(i) $\mathbb{D} / \Gamma$ is a hyperelliptic Riemann surface.

(ii) $\Gamma$ has a prehyperelliptic fundamental polygon having a rotational symmetry of order two.

(iii) $\Gamma$ has a centralhyperelliptic fundamental polygon.

Proof. ((i) $\Longrightarrow($ ii $))$ Assume that $\mathbb{D} / \Gamma$ is a hyperelliptic Riemann surface of genus $g$, i. e. there is a covering $f: \mathbb{D} / \Gamma \rightarrow \widehat{\mathbb{C}}$ (the Riemann sphere) with $2 g+2$ branched points. It follows that there is a Fuchsian group $\Delta$ with signature $(0,[2,2 g+2,2])$ containing $\Gamma$ as an index two subgroup and let $p: \mathbb{D} \rightarrow \mathbb{D} / \Delta=\widehat{\mathbb{C}}$ be the natural projection. The group $\Delta$ admits a canonical presentation:

$$
\left\langle x_{1}, \ldots, x_{2 g+2} ; x_{1}^{2}=\cdots=x_{2 g+2}^{2}=x_{1} \cdots x_{2 g+2}=1\right\rangle .
$$

The group $\Delta$ has a fundamental region $Q$ that is a convex polygon with $2 g+2$ sides, $\lambda_{1}, \ldots, \lambda_{2 g+2}$, and the middle point $m_{i}$ of each side $\lambda_{i}$ is the fixed point of the elliptic generator $x_{i}$, i. e. $Q$ is the canonical fundamental polygon for $\Delta$. The existence of such canonical fundamental polygon for $\Delta$ is a classic result by FrickeKlein (pp. 294-320 of [2] and p. 241 of [3]). The vertices of $Q$ belong to one orbit of $\Delta$. 
In order to be selfcontained we shall sketch a construction of the polygon $Q$. Let $W_{1}, \ldots, W_{2 g+2}$ be the fixed points of the generators $x_{1}, \ldots, x_{2 g+2}$. The points $W_{1}, \ldots, W_{2 g+2}$ do not lie on a hyperbolic line (in the contrary case all the elements of $\Delta$ left invariant such hyperbolic line $\lambda$, but then all the hyperbolic elements in $\Delta$ have $\lambda$ as axis and $\Delta$ cannot contain a surface subgroup). The convex hull $\Pi$ of $W_{1}, \ldots, W_{2 g+2}$ has nonempty interior. Let $\Sigma$ be the set of all hyperbolic lines joining fixed points of elements of $\Delta$. The set $\Sigma$ is countable and then there is a point $W \in \stackrel{\circ}{\Pi}-\cup_{r \in \Sigma} r$. Now there are geodesic segments $\gamma_{1}, \ldots, \gamma_{2 g+2}$ joining $f(W)$ with the branched points of $f$. The result of cutting the orbifold $\mathbb{D} / \Delta$ by $\gamma_{1}, \ldots, \gamma_{2 g+2}$ and the lifting by $p$ produces the fundamental convex polygon $Q$.

Let $\delta_{i}$ be a hyperbolic segment in $Q$ joining $m_{i}$ with $m_{i+1}, i=1, \ldots, 2 g+1$. Each $\delta_{i}$ determines a triangle $T_{i}$ with vertices $m_{i}, m_{i+1}$, and a vertex of $Q$. We define

$$
R=\left(Q-\left(\bigcup_{i=1}^{2 g+1} T_{i}\right)\right) \cup\left(\bigcup_{i=1}^{2 g+1} x_{i} \cdots x_{1}\left(T_{i}\right)\right)
$$

The polygon $R$ is a fundamental region for $\Delta$.

The polygon $R$ has $4 g$ sides:

$$
\delta_{1} \cup \delta_{1}^{\prime}, \delta_{2}, \delta_{3}, \ldots, \delta_{2 g}, \delta_{2 g+1} \cup \delta_{2 g+1}^{\prime}, \delta_{2 g}^{\prime}, \delta_{2 g-1}^{\prime}, \ldots, \delta_{2}^{\prime},
$$

where $\delta_{i}^{\prime}=x_{i} \cdots x_{1}\left(\delta_{i}\right)$.

Let $\widehat{V}_{i}$ (resp. $\widehat{V_{i}^{\prime}}$ ) be the measure of the interior angle with vertex $\delta_{i} \cap \delta_{i+1}$ (resp. $\left.\delta_{i}^{\prime} \cap \delta_{i+1}^{\prime}\right)$. By the construction of $R$ we have that $\widehat{V}_{i}+\widehat{V}_{i}^{\prime}=\pi$.

The polygon $P=R \cup x_{2 g+2}(R)$ is a fundamental region for $\Gamma$ and $x_{2 g+2}$ is a rotational symmetry of order two for $P$. Since $\widehat{V_{2 g+1}}+\widehat{V_{2 g+1}^{\prime}}=\pi$ the polygon $P$ has $8 g-4$ sides. The equalities $\delta_{i}^{\prime}=x_{i} \cdots x_{1}\left(\delta_{i}\right), \widehat{V}_{i}+\widehat{V_{i}^{\prime}}=\pi$ and the fact that $x_{2 g+2}$ is a symmetry imply the prehyperelliptic quality of $P$.

$(($ ii) $\Longrightarrow($ iii) $)$ Let $P$ be a prehyperelliptic fundamental polygon with rotational symmetry $r$ of order two with fixed point $O$. Let $l_{\overline{1}}, \ldots, l_{\bar{m}}$ be the sides of $P$ and $V_{\overline{1}}, \ldots, V_{\bar{m}}$ be the vertices: $l_{\bar{i}} \cap l_{\overline{i+1}}=V_{i}$, satisfying the conditions of Definition 2.1. Let $d_{i}$ be the diagonal of $P$ joining $V_{\bar{i}}$ with $V_{\bar{i}+\frac{m}{2}}$. By the rotational symmetry of $P$, all the diagonals $d_{i}$ intersect in $O$. Let $p: \mathbb{D} \rightarrow \mathbb{D} / \Gamma$ be the natural projection and $S_{2 g}=\left\{p\left(d_{i}\right): i=1, \ldots, 2 g\right\}$. The set $S_{2 g}$ consists of $2 g$ closed geodesics which intersect in $p(O)$ and does not intersect in other point (i. e. $S_{2 g}$ is a $2 g$-star, see the Definition 2.8 below). Cutting $\mathbb{D} / \Gamma$ by $S_{2 g}$ we obtain a centralhyperelliptic fundamental polygon for $\Gamma$.

((iii) $\Longrightarrow$ (i)) Let $C$ be a centralhyperelliptic polygon. Then, by Proposition 2.3, $C$ has a rotational symmetry $r$ of order two. The identifications induced by the elements of $\Gamma$ on the sides of $C$ are compatible with the symmetry $r$. Hence $r$ induces an order two automorphism $\phi$ of $\mathbb{D} / \Gamma$. Let $p: \mathbb{D} \rightarrow \mathbb{D} / \Gamma$ be the natural projection. The number 
of fixed points of $\phi$ is $2 g+2$ : the image by $p$ of the fixed point of $r$, the image by $p$ of the $2 g$ middle points of the sides of $C$ and the point which is the projection of the vertices of $C$ by $p$.

Remark 2.5. From the above proof we have also the following geometrical result: a prehyperelliptic polygon $P$ has rotational symmetry of order two if and only if the diagonals of $P$ joining opposite vertices cut in a point.

We obtain from Theorem 2.4 the characterizations of hyperellipticity given in [4] and $[6]$.

Definition 2.6 (Necklace). A (geodesic) necklace on a Riemann surface of genus $g$ is a cyclically ordered set of $2 g+2$ simple nonseparating closed geodesics $L_{1}, \ldots, L_{2 g+2}$, where each $L_{i}$ intersects $L_{i-1}$ exactly one, intersects $L_{i+1}$ exactly one, and is otherwise disjoint from every other geodesic in the necklace.

Corollary 2.7 (B. Maskit [4]). A Riemann surface $X$ is hyperelliptic if and only if $X$ has an evenly spaced geodesic necklace.

Proof. Assume that $X$ is a hyperelliptic Riemann surface. Then $X=\mathbb{D} / \Gamma$ where $\Gamma$ is a surface Fuchsian group admitting a prehyperelliptic fundamental polygon $P$ with a rotational order two symmetry (Theorem 2.4 (ii)). The sides of the polygon $P$ and the axis of the hyperbolic generators of $\Gamma$ identifying $l_{0}$ with $l_{\frac{m}{2}}$ and $l_{\frac{m}{4}}$ with $l_{\frac{3 m}{4}}$ produce a necklace in $X$. The fact that the polygon $P$ is symmetric implies that the necklace is evenly spaced.

If we have an evenly spaced necklace on $X$, cutting $X$ by such necklace we obtain a prehyperelliptic fundamental polygon for a Fuchsian group uniformizing $X$. The condition of being evenly spaced gives that length $\left(l_{i}\right)=\operatorname{length}\left(l_{i+\frac{m}{2}}\right)$. By a similar argument to the one used in the proof of Proposition 3, it results that the polygon $P$ has a rotational symmetry of order two and then by the Theorem $4, X$ is hyperelliptic.

Definition 2.8 (Star). A (geodesic) $k$-star in a Riemann surface is a set $S_{k}$ of $k$ simple closed geodesics which all intersect in the same point and such that among the elements of $S_{k}$ there are no further intersection points.

Corollary 2.9 (P. S. Schaller [6]). A Riemann surface $X$ is hyperelliptic if and only if $X$ has a $(2 g-2)$-star.

Proof. Assume that $X$ is hyperelliptic. According to Theorem 2.4 (iii), $X$ can be uniformized by a surface Fuchsian group admitting a centralhyperelliptic fundamental polygon. The sides of such polygon produce on $X$ a $2 g$-star which contains, obviously, a $(2 g-2)$-star.

Suppose now that $X$ has a $(2 g-2)$-star $S_{2 g-2}$. Cutting the surface $X$ by $S_{2 g-2}$ we obtain a surface $T_{1}$ homeomorphic to a torus with a boundary component and such boundary component is piecewise geodesic. Since $T_{1}$ is hyperelliptic (all surfaces 
with this topological type are hyperelliptic), let $p_{1}$ and $p_{2}$ be two of the fixed points of the hyperelliptic involution $h$ of $T_{1}$ and $V$ be one of the vertices in $\partial T_{1}$. Let $a_{i}$, $i=1,2$, be two disjoint geodesic arcs joining $V$ with $p_{i}$ and containing no other fixed points of $h$. The set $S_{2 g}=S_{2 g-2} \cup\left\{a_{1} \cup h\left(a_{1}\right)\right\} \cup\left\{a_{2} \cup h\left(a_{2}\right)\right\}$ is a $2 g$-star. Cutting $X$ by $S_{2 g}$ we obtain a centralhyperelliptic fundamental polygon for a surface Fuchsian group uniformizing $X$. Hence, by Theorem 2.4, $X$ is hyperelliptic.

\section{References}

[1] H. M. Farkas and I. Kra, Riemann surfaces, 2nd ed., Graduate Texts in Mathematics, vol. 71 Springer-Verlag, New York, 1992, ISBN 0-387-97703-1.

[2] R. Fricke and F. Klein, Vorlesungen über die Theorie der automorphen Funktionen. Band 1: Die gruppentheoretischen Grundlagen. Band II: Die funktionentheoretischen Ausführungen und die Andwendungen, Bibliotheca Mathematica Teubneriana, Bände 3, vol. 4, Johnson Reprint Corp., New York, 1965.

[3] J. Lehner, Discontinuous groups and automorphic functions, Mathematical Surveys, No. VIII, American Mathematical Society, Providence, R.I., 1964.

[4] B. Maskit, A new characterization of hyperellipticity, Michigan Math. J. 47 (2000), 3-14.

[5] R. Miranda, Algebraic curves and Riemann surfaces, Graduate Studies in Mathematics, vol. 5, American Mathematical Society, Providence, RI, 1995, ISBN 0-8218-0268-2.

[6] P. S. Schaller, Geometric characterization of hyperelliptic Riemann surfaces, Ann. Acad. Sci. Fenn. Math. 25 (2000), 25-90. 